TRANSACTIONS OF THE

AMERICAN MATHEMATICAL SOCIETY

Volume 348, Number 2, February 1996

\title{
HARMONIC BERGMAN FUNCTIONS ON HALF-SPACES
}

\author{
WADE C. RAMEY AND HEUNGSU YI
}

\begin{abstract}
We study harmonic Bergman functions on the upper half-space of $\mathbf{R}^{n}$. Among our main results are: The Bergman projection is bounded for the range $1<p<\infty$; certain nonorthogonal projections are bounded for the range $1 \leq p<\infty$; the dual space of the Bergman $L^{1}$-space is the harmonic Bloch space modulo constants; harmonic conjugation is bounded on the Bergman spaces for the range $1 \leq p<\infty$; the Bergman norm is equivalent to a "normal derivative norm" as well as to a "tangential derivative norm".
\end{abstract}

\section{INTRODUCTION}

The upper half-space $H=H_{n}$ is the open subset of $\mathbf{R}^{n}$ given by

$$
H=\left\{(x, y) \in \mathbf{R}^{n}: y>0\right\},
$$

where we have written a typical point $z \in \mathbf{R}^{n}$ as $z=(x, y)$, with $x \in \mathbf{R}^{n-1}$ and $y \in \mathbf{R}$. Let $p \in[1, \infty)$. We are interested in harmonic functions $u$ on $H$ such that

$$
\|u\|_{p}=\left(\int_{H}|u|^{p} d V\right)^{1 / p}<\infty
$$

Here $d V$ denotes Lebesgue volume measure on $H$, which we may also write as $d z$, $d w$, etc. The collection of all such functions will be denoted $b^{p}=b^{p}(H)$. We refer to the Banach spaces $b^{p}$ as harmonic Bergman spaces. (That they are Banach spaces is proved in Proposition 8.3 of [4].)

Harmonic Bergman spaces have not been studied as extensively as their holomorphic counterparts. One property of the holomorphic Bergman spaces that we will sorely miss is their invariance under multiplication by bounded holomorphic functions; nothing comparable holds for harmonic Bergman spaces. Furthermore, most work on Bergman spaces - even in the holomorphic case - has been done for bounded domains. As we will see, the unboundedness of $H$ causes some difficulties; for example, the Bergman kernel is not integrable over $H$. Thus, unlike the case of the disk or ball, the Bergman projection of an $L^{\infty}$-function on $H$ is not even defined. Finding the dual space of $b^{1}$ —not an easy task even in the classical settings - will therefore involve some extra work. Another problem we have on $H$ is that of finding "tame" dense subspaces of $b^{p}$ (especially when $p=1$ ). On the ball, of course, we can always choose the space of harmonic polynomials. On $H$ it is not so clear what to do; finding dense subspaces in this context will be a recurrent theme throughout the paper.

Received by the editors October 13, 1994.

1991 Mathematics Subject Classification. Primary 31B05; Secondary 31B10, 30D55, 30D45.

Key words and phrases. Bergman kernel, projection operators, dual spaces, harmonic Bloch space.

(C)1996 American Mathematical Society 
Fortunately, not all the news about $H$ is bad. For example, $H$ is invariant under both horizontal translations and dilations - transformations that preserve harmonic functions. (The horizontal translations on $H$ correspond to rotations on the unit ball, but there is nothing on the ball analogous to the dilation structure of $H$.) Moreover, $H$ is a product domain, giving us a way to integrate by parts that will be crucial for many of our results.

Let us summarize the main results of this paper. Section 2 is devoted to introductory material and some basic properties and examples. In Section 3 we show that the Bergman projection, initially defined as the orthogonal projection of $L^{2}$ onto $b^{2}$, extends to a bounded projection of $L^{p}$ onto $b^{p}$ for the range $1<p<\infty$. Letting $q$ denote the index conjugate to $p$, we then easily show that the dual space of $b^{p}$ can be identified with $b^{q}$ for this range of $p$-values. (The dilation structure of $H$ allows for an easier proof than for the analogous result on, say, the unit disk.)

In Section 4 we study certain nonorthogonal projections of $L^{p}$ onto $b^{p}$. These projections are in many ways better behaved than is the Bergman projection, and they will be useful in many contexts. For example, they allow us to see that the Bergman norm can be reformulated in terms of derivatives. The latter property gives us a nice way to see that the harmonic Bloch space is the appropriate limit of $b^{p}$ as $p \rightarrow \infty$.

As suggested above, finding the dual space of $b^{1}$ is problematic. In Section 5 we will modify the Bergman projection, obtaining an appropriate bounded linear map of $L^{\infty}$ into the harmonic Bloch space. We then prove that the dual space of $b^{1}$ is the harmonic Bloch space modulo constants. The proviso "modulo constants" arises because of the $b^{1}$-cancellation property: If $u \in b^{1}$, then

$$
\int_{H} u(w) d w=0,
$$

as we show in Section 2 .

The last section is devoted to harmonic conjugation. Recall that harmonic conjugation is bounded in the Hardy-space $L^{p}$-norm when $1<p<\infty$, but not when $p=1$. However, for the unit disk and other settings, it is well-known that harmonic conjugation is bounded in the Bergman-space $L^{p}$-norm for all $p \in[1, \infty)$. We prove the analogous result for the upper-half space in all dimensions: If $u \in b^{p}$, with $p \in[1, \infty)$, then there exist unique harmonic conjugates $v_{1}, \ldots, v_{n-1}$ of $u$ that belong to $b^{p}$ (with appropriate norm-bounds). Recalling that Bergman-space norms can be restated in terms of derivatives, we use the conjugation results to show that the $b^{p}$-norm is equivalent to a "tangential derivative" norm as well as to a "normal derivative" norm. A similar result holds for the Bloch-norm. These last results were unanticipated by us and appear to be new (even in the context of the unit disk).

Certain elementary results in this paper, such as the $b^{1}$-cancellation property mentioned above, may be well-known to some readers or appear elsewhere in the literature; we have included them for the sake of completeness and the convenience of the reader.

\section{BASIC PROPERTIES AND EXAMPLES}

Unless otherwise stated, the full range $1 \leq p<\infty$ is intended when discussing $b^{p}$, and all functions are assumed to be complex valued. Any unexplained notation will be as in [4]. 
For an open $\Omega \subset \mathbf{R}^{n}$, we let $b^{p}(\Omega)$ denote the collection of harmonic functions $u$ on $\Omega$ such that

$$
\|u\|_{b^{p}(\Omega)}=\left(\int_{\Omega}|u|^{p} d V\right)^{1 / p}<\infty .
$$

The standard estimate obtained from the mean-value property for harmonic functions shows that if $u \in b^{p}(\Omega)$, then

$$
|u(z)| \leq \frac{1}{V(B)^{1 / p}} \frac{\|u\|_{b^{p}(\Omega)}}{d(z, \partial \Omega)^{n / p}}
$$

for all $z \in \Omega$, where $B$ denotes the unit ball in $\mathbf{R}^{n}$ and $d$ denotes euclidean distance. Inequality (2.1) implies that convergence in the $b^{p}(\Omega)$-norm implies uniform convergence on compact subsets of $\Omega$, that $b^{p}(\Omega)$ is a Banach space, and that for fixed $z \in \Omega$, the map $z \rightarrow u(z)$ is a bounded linear functional on $b^{p}(\Omega)$. (For these and related results see [4], Chapter 8.)

Cauchy's estimates lead from (2.1) to the inequality

$$
\left|D^{\alpha} u(z)\right| \leq C \frac{\|u\|_{b^{p}(\Omega)}}{d(z, \partial \Omega)^{n / p+|\alpha|}},
$$

where $\alpha$ is a multi-index and $C$ is a constant that depends only on $n, p$, and $\alpha$. (We will follow the practice of allowing $C$ to denote a constant whose value may change from line to line; at appropriate places we will specify the parameters on which $C$ depends.)

For most of the paper we are concerned with the setting $\Omega=H$. Here we write $b^{p},\|u\|_{p}$, and $L^{p}$ in place of $b^{p}(H),\|u\|_{b^{p}(H)}$, and $L^{p}(H, d V)$. Note that if $u \in b^{p}$, then (2.2) becomes

$$
\left|D^{\alpha} u(x, y)\right| \leq C \frac{\|u\|_{p}}{y^{n / p+|\alpha|}},
$$

for all $(x, y) \in H$.

For $u$ a function on $H$ and $\delta>0$, let $\tau_{\delta} u$ denote the function on $H$ defined by

$$
\tau_{\delta} u(z)=u(z+(0, \delta)) .
$$

We will need the following fact: If $u \in b^{p}$, then $\tau_{\delta} u \rightarrow u$ in the norm of $b^{p}$ as $\delta \rightarrow 0$. (Proof: If $f \in L^{p}$, then $\tau_{\delta} f \rightarrow f$ in $L^{p}$ as $\delta \rightarrow 0$, as we see by proving this first for continuous $f$ with compact support.)

Taking $\alpha=0$ in (2.3) shows that if $u \in b^{p}$, then $u$ is bounded on each proper half-space contained in $H$, hence is the Poisson integral of its boundary values on each such half-space. In other words,

$$
\tau_{\delta} u=P[u(\cdot, \delta)]
$$

on $H$ for each $\delta>0$. Here

$$
P[f](z)=\int_{\partial H} P(z, s) f(s) d s
$$

denotes the Poisson integral of $f$ (defined for any $f \in L^{p}(\partial H), 1 \leq p \leq \infty$ ), where $d s$ denotes Lebesgue measure on $\partial H$ (which we may also denote by $d x$, etc.). The function $P$ is the Poisson kernel for $H$, defined for $(z, s) \in H \times \partial H$ by

$$
P(z, s)=\frac{2}{n V(B)} \frac{y}{\left(|x-s|^{2}+y^{2}\right)^{n / 2}},
$$


where $z=(x, y)$. Recall that $\int_{\partial H} P(z, s) d s=1$ for all $z \in H$, and that $P(\cdot, s)$ is positive and harmonic on $H$ for each $s \in \partial H$.

The Poisson integral gives us a nice way to derive an important property of Bergman functions.

Theorem 2.1. If $u \in b^{p}$, then the integrals $\int_{\partial H}|u(x, y)|^{p} d x$ increase as $y d e-$ creases.

Proof. Suppose $0<y_{1}<y_{2}$. Then

$$
u\left(x, y_{2}\right)=P\left[u\left(\cdot, y_{1}\right)\right]\left(x, y_{2}-y_{1}\right) .
$$

Applying Jensen's inequality, we get

$$
\left|u\left(x, y_{2}\right)\right|^{p} \leq P\left[\left|u\left(\cdot, y_{1}\right)\right|^{p}\right]\left(x, y_{2}-y_{1}\right) .
$$

Integrating with respect to $x$ and then using Fubini's theorem yields (recall that $\left.\int_{\partial H} P(z, s) d s=1\right)$

$$
\int_{\partial H}\left|u\left(x, y_{2}\right)\right|^{p} d x \leq \int_{\partial H}\left|u\left(x, y_{1}\right)\right|^{p} d x
$$

as desired.

As the proof shows, the conclusion of Theorem 2.1 holds if we only assume that $u$ equals the Poisson integral of its boundary values on every proper half-space contained in $H$.

Note that Theorem 2.1 implies that if $u \in b^{p}$, then $\tau_{\delta} u \in h^{p}=h^{p}(H)$ for every $\delta>0$, where $h^{p}$ is the Hardy $L^{p}$-space of functions $v$ harmonic on $H$ such that

$$
\|v\|_{h^{p}}=\sup _{y>0}\left(\int_{\partial H}|v(x, y)|^{p} d x\right)^{1 / p}<\infty .
$$

We now come to the $b^{1}$ cancellation property mentioned in the introduction: If $u \in b^{1}$, then $\int_{H} u(w) d w=0$. This is implied by the next theorem.

Theorem 2.2. If $u \in b^{1}$, then

$$
\int_{\partial H} u(x, y) d x=0
$$

for every $y>0$.

Proof. Theorem 2.1 shows that $u(\cdot, y) \in L^{1}(\partial H)$ for every $y>0$. Fix $\delta>0$. Then $\tau_{\delta} u=P[u(\cdot, \delta)]$, so that

$$
\begin{aligned}
\int_{H} \tau_{\delta} u(w) d w & =\int_{0}^{\infty} \int_{\partial H} \int_{\partial H} P((x, y), s) u(s, \delta) d s d x d y \\
& =\int_{0}^{\infty} \int_{\partial H} u(s, \delta) d s d y
\end{aligned}
$$

where we have used Fubini's theorem and the fact that $\int_{\partial H} P((x, y), s) d x=1$. Because the inner integral in the last line is independent of $y$, we must have

$$
\int_{\partial H} u(s, \delta) d s=0
$$

as desired. 
Positive functions in $b^{p}$. Unlike the case of the ball, certain $b^{p}$-spaces on $H$ do not contain any positive functions.

Theorem 2.3. The space $b^{p}$ contains a positive function if and only if $p>n /(n-1)$.

Proof. Setting $u(z)=P(z, 0)$, we can easily check that for any $\delta>0$, the positive function $\tau_{\delta} u$ belongs to $b^{p}$ for all $p>n /(n-1)$.

Conversely, suppose $u$ is positive and harmonic on $H$ and that $u \in b^{p}$. By Theorem 7.24 in [4],

$$
u(x, y)=c y+P[\mu](x, y)
$$

for all $(x, y) \in H$, where $c$ is a nonnegative constant and $\mu$ is a positive Borel measure on $\partial H$ such that

$$
\int_{\partial H} \frac{d \mu(s)}{(1+|s|)^{n}}<\infty
$$

We clearly have $c=0$, or else $u$ is not in $b^{p}$. Because $\mu$ is not the zero measure, we can choose a compact set $K \subset \partial H$ such that $\mu(K)>0$. We then easily see that

$$
u(z)>\frac{2}{n V(B)} \frac{y}{(|z|+R)^{n}} \mu(K)
$$

on $H$, where $R=\max \{|s|: s \in K\}$. Thus, because $u \in b^{p}$, we must have $p>$ $n /(n-1)$.

Distinctness of the spaces $b^{p}$. We next show that on $H$, no Bergman space is properly contained in another. (This does not seem as simple to prove as the analogous result for Hardy spaces on $H$, which is obvious from the Poisson integral representation.)

Theorem 2.4. If $p \neq q$, then $b^{p}$ does not contain $b^{q}$.

Proof. Suppose to the contrary that $b^{p} \subset b^{q}$. Because convergence in any Bergman space implies uniform convergence on compact subsets, the closed-graph theorem shows then that the identity map from $b^{p}$ to $b^{q}$ is continuous. Thus there exists a constant $C$ such that

$$
\|u\|_{q} \leq C\|u\|_{p}
$$

for all $u \in b^{p}$.

To show that the last inequality fails, we consider first the case $n>2$. With $z=(x, y)$, define

$$
u(z)=D_{y}^{3}|z|^{2-n}
$$

for $z \in H$. Being a partial derivative of the harmonic function $|z|^{2-n}, u$ is harmonic (on $\mathbf{R}^{n} \backslash\{0\}$ ). It is easy to see that

$$
u(z)=\frac{f(z)}{|z|^{n+4}}
$$

for some homogeneous polynomial $f$ of degree 3 . (Note: The polynomial $f$ is itself harmonic, but we do not need this fact here.)

For any $\delta>0$, we use homogeneity to see that

$$
\begin{aligned}
\left\|\tau_{\delta} u\right\|_{p}^{p} & =\int_{H} \frac{|f(z+(0, \delta))|^{p}}{|z+(0, \delta)|^{(n+4) p}} d z \\
& =\frac{\delta^{n+3 p}}{\delta^{(n+4) p}} \int_{H} \frac{|f(z+(0,1))|^{p}}{|z+(0,1)|^{(n+4) p}} d z
\end{aligned}
$$


Thus, $\left\|\tau_{\delta} u\right\|_{p}=C(n, p) \delta^{(n / p)-n-1}$, which implies

$$
\frac{\left\|\tau_{\delta} u\right\|_{q}}{\left\|\tau_{\delta} u\right\|_{p}}=C(n, p, q) \delta^{n / q-n / p}
$$

for all $\delta>0$. Because $p \neq q$, the right side above is not a bounded function of $\delta$. Thus (2.4) fails, and therefore $b^{p}$ is not contained in $b^{q}$, as we wanted to show.

The proof when $n=2$ is the same, except that the function $|z|^{2-n}$ should be replaced by $\log |z|$.

Derivatives of Bergman functions. If $\alpha$ is a multi-index, $\alpha \neq 0$, then there exist functions $u \in b^{p}$ for which $D^{\alpha} u \notin b^{p}$. (We can prove this with the closed-graph theorem by an argument similar to the one just given; exhibiting such a function explicitly is not trivial.) This phenomenon occurs because of possible bad behavior of $D^{\alpha} u$ near $\partial H$-not near $\infty$-as the next result shows.

Theorem 2.5. Let $\Omega$ be a half-space properly contained in $H$. If $u \in b^{p}$, then $D^{\alpha} u \in b^{p}(\Omega)$ for every $\alpha$.

Proof. Let $\delta>0$, set $\Omega=\left\{z \in H: z_{n}>\delta\right\}$, and assume $u \in b^{p}$. Fix a multi-index $\alpha$. For $z \in \Omega$, let $B_{z}$ denote the open ball with center $z$ and radius $\delta$; note that $B_{z} \subset H$. By $(2.2)$ we have

$$
\left|D^{\alpha} u(z)\right| \leq \frac{C}{\delta^{n / p+|\alpha|}}\|u\|_{b^{p}\left(B_{z}\right)}
$$

where $C=C(n, \alpha, p)$. For $(z, w) \in \Omega \times H$, set $\chi(z, w)=1$ if $|z-w|<\delta$ and $\chi(z, w)=0$ otherwise. Then by Fubini's theorem we have

$$
\begin{aligned}
\int_{\Omega}\left|D^{\alpha} u(z)\right|^{p} d z & \leq \frac{C^{p}}{\delta^{n+|\alpha| p}} \int_{\Omega} \int_{H}|u(w)|^{p} \chi(z, w) d w d z \\
& \leq \frac{C^{p}}{\delta^{n+|\alpha| p}}\|u\|_{p}^{p} V(B) \delta^{n},
\end{aligned}
$$

giving the conclusion of the theorem.

The next result shows how differently Bergman functions on $H$ behave when compared with Bergman functions on bounded domains.

Theorem 2.6. If $u \in b^{p}$ and $\alpha$ is a multi-index, then $D^{\alpha} u \equiv 0$ if and only if $u \equiv 0$.

Proof. We clearly need only worry about the direction " $\Rightarrow$ ", which we prove by induction on $|\alpha|$; note that there is nothing to prove when $\alpha=0$. Assuming this direction has been proved for multi-indices of order $m \geq 0$, suppose that

$$
D_{j} D^{\alpha} u \equiv 0
$$

where $|\alpha|=m$. Then $D^{\alpha} u$ is independent of the $j^{\text {th }}$ coordinate variable.

Let $\Omega$ be any half-space properly contained in $H$. By Theorem 2.5, $D^{\alpha} u \in b^{p}(\Omega)$. But Fubini's theorem shows that if $v \in b^{p}(\Omega)$ and $v$ is independent of one of the coordinate variables, then $v \equiv 0$. We conclude that $D^{\alpha} u \equiv 0$ on $\Omega$, which implies $D^{\alpha} u \equiv 0$ on $H$. By our induction hypothesis, $u \equiv 0$, as desired. 


\section{The Bergman PRojection}

The easiest Bergman space to understand is $b^{2}$. This is because $b^{2}$ is a Hilbert space, so that we have the entire Hilbert-space machinery at our disposal.

Fix $z \in H$. Because point evaluation is a bounded linear functional on $b^{2}$, there exists a unique function $R(z, \cdot) \in b^{2}$ such that

$$
u(z)=\int_{H} u(w) \overline{R(z, w)} d w
$$

for all $u \in b^{2}$. The function $R(z, w)$ is called the reproducing kernel or the Bergman kernel for $b^{2}$. Standard results (see Chapter 8 in [4]) show that $R(z, w)=R(w, z)$ and that $R(z, w)$ is real valued; thus we can remove the complex conjugate in the integral above.

Because $b^{2}$ is a closed subspace of the Hilbert space $L^{2}$, there is a unique orthogonal projection $\Pi$ of $L^{2}$ onto $b^{2}$. We call $\Pi$ the Bergman projection. It is easy to check that

$$
\Pi f(z)=\int_{H} f(w) R(z, w) d w
$$

for all $f \in L^{2}$. From (3.1) we obtain

$$
\int_{H}(\Pi f) g d V=\int_{H} f(\Pi g) d V
$$

for all $f, g \in L^{2}$.

By Theorem 8.22 in [4],

$$
R(z, w)=\frac{4}{n V(B)} \frac{n\left(z_{n}+w_{n}\right)^{2}-|z-\bar{w}|^{2}}{|z-\bar{w}|^{n+2}},
$$

where $\bar{w}=\left(w_{1}, \ldots, w_{n-1},-w_{n}\right)$. From this formula we see that

$$
|R(z, w)| \leq \frac{C}{|z-\bar{w}|^{n}}
$$

for all $z, w \in H$, where $C=C(n)$.

The last estimate shows, after integrating in polar coordinates, that $R(z, \cdot) \in b^{q}$ for all $q \in(1, \infty]$. Thus the integral in (3.1) is well defined whenever $f \in L^{p}$ for $p \in[1, \infty)$. Extending the definition of $\Pi$ to every $L^{p}, 1 \leq p<\infty$, we see that $\Pi f$ is a harmonic function on $H$ whenever $f$ belongs to one of these $L^{p}$-spaces.

We next claim that $\Pi$ is the identity on $b^{p}$, i.e., that

$$
u(z)=\int_{H} u(w) R(z, w) d w
$$

holds for all $u \in b^{p}$ and for all $p \in[1, \infty)$. Proving this would be easy if we knew that $b^{p} \cap b^{2}$ is dense in $b^{p}$. While this is true (and will follow as a corollary of results we obtain later), we do not know an elementary proof. Fortunately, the proof of Theorem 8.22 in [4] can be used nearly word for word to prove (3.4); we omit the argument here. (It resembles several arguments we give below.) We now show that for $1<p<\infty, \Pi$ is a bounded projection from $L^{p}$ onto $b^{p}$. The following lemma will be useful in proving this.

Lemma 3.1. If $1<p<\infty$, then there is a constant $C=C(n, p)$ such that

$$
\int_{H} \frac{w_{n}^{-1 / p}}{|z-\bar{w}|^{n}} d w=C z_{n}^{-1 / p}
$$

for all $z \in H$. 
Proof. Fix $z \in H$. Letting $w=(x, y)$, we have

$$
\int_{H} \frac{y^{-1 / p}}{|z-\bar{w}|^{n}} d w=\int_{0}^{\infty} \frac{y^{-1 / p}}{z_{n}+y} \int_{\partial H} \frac{z_{n}+y}{|z-\bar{w}|^{n}} d x d y .
$$

Note that the inner integral in the right side of (3.5) equals

$$
\frac{n V(B)}{2} \int_{\partial H} P(z+(0, y), x) d x=\frac{n V(B)}{2},
$$

where $P$ is the Poisson kernel for $H$. Hence, after applying change of variable $y \longmapsto z_{n} y$, we see that the right side of (3.5) equals $C z_{n}^{-1 / p}$, where

$$
C=\frac{n V(B)}{2} \int_{0}^{\infty} \frac{y^{-1 / p}}{1+y} d y<\infty .
$$

This completes the proof.

Theorem 3.2. If $1<p<\infty$, then $\Pi$ is a bounded projection of $L^{p}$ onto $b^{p}$.

Proof. We only need to check the boundedness of $\Pi$. Let $f \in L^{p}$. Then for $z \in H$, (3.3) implies

$$
\begin{aligned}
|\Pi f(z)| & \leq C \int_{H}|f(w)| \frac{1}{|z-\bar{w}|^{n}} d w \\
& =C \int_{H}|f(w)| y^{1 / p q} \frac{1}{|z-\bar{w}|^{n}} y^{-1 / p q} d w,
\end{aligned}
$$

where $q$ denotes the index conjugate to $p$ and $w=(x, y)$. Applying Hölder's inequality to the functions

$$
|f(w)| \frac{y^{1 / p q}}{|z-\bar{w}|^{n / p}}, \frac{y^{-1 / p q}}{|z-\bar{w}|^{n / q}}
$$

we have

$$
|\Pi f(z)|^{p} \leq C\left(\int_{H}|f(w)|^{p} \frac{y^{1 / q}}{|z-\bar{w}|^{n}} d w\right)\left(\int_{H} \frac{y^{-1 / p}}{|z-\bar{w}|^{n}} d w\right)^{p / q} .
$$

Hence, from Lemma 3.1, we get

$$
\int_{H}|\Pi f(z)|^{p} d z \leq C \int_{H} \int_{H}|f(w)|^{p} \frac{y^{1 / q}}{|z-\bar{w}|^{n}} d w z_{n}^{-1 / q} d z .
$$

By applying Fubini's Theorem and then Lemma 3.1 once again, we have

$$
\int_{H}|\Pi f(z)|^{p} d z \leq C \int_{H}|f(w)|^{p} d w,
$$

as desired. (Here $C=C(n, p)$.)

Remark. In the proof of the last theorem, we have used ideas that can be found [3] and [12], which originate in [6] and [9]. The dilation structure of $H$ allows for a simpler implementation of these ideas than in the case of the unitdisk. 
Although $\Pi$ is the identity operator on $b^{1}$, it does not map $L^{1}$ into $b^{1}$. To see this, let $f$ denote the characteristic function of some small ball centered at $(0,1)$, divided by the volume of this ball. By the mean value property for harmonic functions,

$$
\Pi f(z)=R(z,(0,1)) .
$$

In a thin cone centered along the $y$-axis,

$$
|R(z,(0,1))| \approx \frac{1}{1+|z|^{n}},
$$

which shows that $\Pi f$ is not in $b^{1}$.

The dual space of $b^{p}$ for $1<p<\infty$. We now prove that for $1<p<\infty$, the dual space of the harmonic Bergman space $b^{p}$ can be identified with $b^{q}$, where $q$ denotes the index conjugate to $p$. It is clear that if $v \in b^{q}$, then the mapping

$$
u \longmapsto \int_{H} u v d V
$$

is a bounded linear functional on $b^{p}$ whose norm is at most $\|v\|_{q}$. Furthermore, distinct functions in $b^{q}$ induce distinct linear functionals in $b^{p}$. To see this, suppose $v \in b^{q}$ and that $v$ induces the zero-functional on $b^{p}$. Because $R(z, \cdot) \in b^{p}$, we have

$$
v(z)=\int_{H} R(z, w) v(w) d w=0
$$

for every $z \in H$ by (3.4). I.e., $v \equiv 0$ as desired.

Therefore, to prove our duality result, we only need to show that every bounded linear functional on $b^{p}$ is of the form (3.6), with an appropriate estimate on $\|v\|_{q}$.

Theorem 3.3. If $1<p<\infty$, then $\left(b^{p}\right)^{*} \cong b^{q}$, where $q$ denotes the index conjugate to $p$.

Proof. We first show

$$
\int_{H}(\Pi f) g d V=\int_{H} f(\Pi g) d V
$$

for all $f \in L^{p}$ and for all $g \in L^{q}$. Note that (3.7) is true whenever $p=q=2$. Since $L^{2} \cap L^{r}$ is dense in $L^{r}$ for every $r \in(1, \infty),(3.7)$ follows from the $p=2$ case by a simple limiting argument using Hölder's inequality and Theorem 3.2.

Now suppose $\Lambda$ is a bounded linear functional on $b^{p}$. By the Hahn-Banach Theorem, there is a function $f \in L^{q}$ such that $\|\Lambda\|=\|f\|_{q}$ and

$$
\Lambda(u)=\int_{H} u f d V
$$

for all $u \in b^{p}$. Because $u=\Pi u$ for all $u \in b^{p}$, we use (3.7) to conclude that

$$
\Lambda u=\int_{H} u(\Pi f) d V
$$

for all $u \in b^{p}$. Thus $\Lambda$ is induced by the $b^{q}$-function $\Pi f$. Finally, $\|\Pi f\|_{q} \leq C\|f\|_{q}=$ $C\|\Lambda\|$, where $C=C(n, q)$. This, together with the remarks made at the beginning of this subsection, completes the proof. 
Dense subspaces of $b^{p}$ for $1<p<\infty$. It is sometimes useful to know that there is a dense subspace of $b^{p}$ consisting of functions that vanish to high order at $\infty$. For this purpose, let $\alpha$ be a multi-index and set

$$
\mathcal{M}_{\alpha}=\operatorname{span}\left\{D^{\alpha} R(z, w): z \in H\right\}
$$

where "span" denotes the linear span over C. (We are thinking of $D^{\alpha} R(z, w)$ as a function of $w$.) Because

$$
D_{w}^{\alpha} R(z, w)=(-1)^{\alpha_{1}+\cdots+\alpha_{n-1}} D_{z}^{\alpha} R(z, w),
$$

where $\alpha=\left(\alpha_{1}, \ldots, \alpha_{n}\right)$, we need not specify the variable of differentiation in the definition of $\mathcal{M}_{\alpha}$.

To estimate the size of the derivatives of $R(z, w)$, observe that

$$
D_{z}^{\alpha} R(z, w)=\frac{f_{\alpha}(z-\bar{w})}{|z-\bar{w}|^{n+2+2|\alpha|}}
$$

where $f_{\alpha}$ is a homogeneous polynomial of degree $2+|\alpha|$; this is straightforward to verify by induction. Therefore

$$
\left|D_{z}^{\alpha} R(z, w)\right| \leq \frac{C(n, \alpha)}{|z-\bar{w}|^{n+|\alpha|}}
$$

for all $z, w \in H$.

From (3.8) we see that if $u \in \mathcal{M}_{\alpha}$, then there is a constant $C$ such that

$$
|u(w)| \leq C \frac{1}{(1+|w|)^{n+|\alpha|}}
$$

for all $w \in H$. Thus the next theorem, which is a corollary of the duality result obtained above, gives us dense subspaces of $b^{p}$ whose members vanish at $\infty$ to high order.

Theorem 3.4. Each $\mathcal{M}_{\alpha}$ is dense in $b^{p}$ for $1<p<\infty$.

Proof. Fix $\alpha$ and $1<p<\infty$. Suppose $v \in b^{q}$ satisfies

$$
\int_{H} u v d V=0
$$

for all $u \in \mathcal{M}_{\alpha}$. Then

$$
\int_{H} D_{z}^{\alpha} R(z, w) v(w) d w=D_{z}^{\alpha} \int_{H} R(z, w) v(w) d w=D^{\alpha} v(z)=0
$$

for all $z \in H$. This implies that $v \equiv 0$ by Theorem 2.6. Hence by a standard corollary of the Hahn-Banach Theorem and Theorem 3.3, $\mathcal{M}_{\alpha}$ must be dense in $b^{p}$.

Interestingly, the space $\mathcal{M}_{\alpha}$ is not always dense in $b^{1}$. Note that $\mathcal{M}_{0}$ is not even contained in $b^{1}$, while $\mathcal{M}_{\alpha}$ is contained in $b^{1}$ if $\alpha \neq 0$. As we show later, if $\alpha \neq 0$, a difference arises between the cases $n>2$ and $n=2$ : When $n>2, \mathcal{M}_{\alpha}$ is not dense in $b^{1}$ for most choices of $\alpha$; when $n=2, \mathcal{M}_{\alpha}$ is always dense in $b^{1}$.

We next show that functions in $b^{p}$ can be approximated on $H$ by $b^{p}$-functions defined on much larger half-spaces. For this purpose, set

$$
H_{\delta}=\left\{(x, y) \in \mathbf{R}^{n}: y>-\delta\right\}
$$

for $\delta>0$.

Theorem 3.5. If $1<p<\infty$, then $b^{p}\left(H_{\delta}\right)$ is dense in $b^{p}$ for each $\delta>0$. 
Proof. Fix $\delta>0$. Because $R(z, \cdot) \in b^{p}$, we easily see that $R(z+(0, \delta), \cdot) \in b^{p}\left(H_{\delta}\right)$ for any $z \in H$. Now suppose $v \in b^{q} \cong\left(b^{p}\right)^{*}$ and that $v$ vanishes on $b^{p}\left(H_{\delta}\right)$. Then

$$
v(z+(0, \delta))=\int_{H} R(z+(0, \delta), w) v(w) d w=0
$$

for all $z \in H$, which implies $v \equiv 0$ on $H$. By a standard corollary of the HahnBanach Theorem, $b^{p}\left(H_{\delta}\right)$ must be dense in $b^{p}$.

Remark. After obtaining the dual space of $b^{1}$ in Section 5 , we will be able to show that $b^{1}\left(H_{\delta}\right)$ is dense in $b^{1}$ for every $\delta>0$. Note the contrast with the Hardy spaces $h^{p}$ : If $1<p<\infty$, then $h^{p}\left(H_{\delta}\right)$ is dense in $h^{p}$, while this fails if $p=1$ (there is nothing in $h^{1}\left(H_{\delta}\right)$ close to $P(\cdot, 0)$, because the latter is the Poisson integral of a singular measure).

\section{Nonorthogonal PROJECTIONS}

In this section, we study certain nonorthogonal projections of $L^{p}$ onto $b^{p}$ for $p \in[1, \infty$ ). (We call them "nonorthogonal" because they are not orthogonal when acting on the Hilbert-space $L^{2}$.) Unlike the Bergman projection, these projections are bounded on $L^{1}$. The $L^{1}$-boundedness will enable us, finally, to obtain dense subspaces of $b^{1}$ whose members vanish to high order at $\infty$; this will be useful in finding the dual space of $b^{1}$ (Section 5). Additionally, the new projections lead to certain "derivative norms" on the Bergman spaces; they will also play a role in the harmonic conjugation results of Section 6 .

Let $m$ denote a nonnegative integer and let $p \in[1, \infty)$. For $f \in L^{p}$ and $z \in H$, define

$$
\Pi_{m} f(z)=c_{m} \int_{H} f(w) y^{m} D_{y}^{m} R(z, w) d w,
$$

where $c_{m}=(-2)^{m} / m$ ! and $w=(x, y)$. Note that by $(3.8), y^{m} D_{y}^{m} R(z, \cdot) \in b^{q}$ for all $q \in(1, \infty]$, so that the above integral is well-defined and harmonic on $H$ for all $f \in L^{p}, 1 \leq p<\infty$. Note also that $\Pi_{0}$ is the Bergman projection $\Pi$. We study $\Pi_{m}$ by exploiting the connection between the Bergman and Poisson kernels. For $z, w \in H$, the "extended Poisson kernel" is the function

$$
P(z, w)=\frac{2}{n V(B)} \frac{z_{n}+w_{n}}{|z-\bar{w}|^{n}}
$$

Note that $P(z,(x, y))=P(z+(0, y), x)$ for all $z,(x, y) \in H$, which implies $P(\cdot, w)$ is a positive harmonic function on $\left\{z \in \mathbf{R}^{n}: z_{n}>-w_{n}\right\}$ for each $w \in H$. Furthermore, by (3.2),

$$
R(z, w)=-2 \frac{\partial P(z, w)}{\partial w_{n}}
$$

for all $z, w \in H$. Thus the definition of $\Pi_{m}$ can be rewritten

$$
\Pi_{m} f(z)=-2 c_{m} \int_{H} f(w) y^{m} D_{y}^{m+1} P(z, w) d w .
$$

Theorem 4.2 below is the main result of this section. We first prove the following lemma, which contains an identity we need in proving Theorem 4.2.

Lemma 4.1. Let $1 \leq p<\infty$ and let $\delta>0$. If $u \in b^{p}\left(H_{\delta}\right)$, then

$$
u(z)=-2 c_{m} \int_{H}\left[D_{y}^{m+1} u(w)\right] y^{m} P(z, w) d w
$$

for all $z \in H .($ Here $w=(x, y)$. 
Proof. Fix $z \in H$. Because $u \in b^{p}\left(H_{\delta}\right)$, we have

$$
\left|D^{\alpha} u(x, y)\right| \leq \frac{C}{(y+\delta)^{n / p+|\alpha|}}
$$

on $H$ for every $\alpha$. This estimate guarantees that the integrand in (4.2) belongs to $L^{1}$. It also shows that $D_{y}^{m+1} u$ is bounded on $H$, so that we may apply the Poisson integral formula. Thus the integral in (4.2) equals

$$
\begin{gathered}
\int_{0}^{\infty} y^{m} \int_{\partial H} D_{y}^{m+1} u(x, y) P(z+(0, y), x) d x d y \\
=\int_{0}^{\infty} y^{m} D_{y}^{m+1} u(z+(0,2 y)) d y .
\end{gathered}
$$

Integrating by parts $m$ times, we see that the last term equals

$$
\frac{1}{c_{m}} \int_{0}^{\infty} D_{y} u(z+(0,2 y)) d y=\frac{1}{-2 c_{m}} u(z) .
$$

This completes the proof.

Remark. The idea of integrating by parts in the manner of the above proof - an idea we will use many times in this paper - is inspired by the proof of Theorem 8.22 in [4].

Theorem 4.2. If $1 \leq p<\infty$ and $m>0$, then $\Pi_{m}$ is a bounded projection of $L^{p}$ onto $b^{p}$.

Proof. We first show, by induction on $m$, that $\Pi_{m}$ is the identity operator on $b^{p}$. (Recall that $\Pi_{0}$ is the Bergman projection, so that $\Pi_{0}$ is the identity on $b^{p}$.) Assuming that $\Pi_{m-1}$ is the identity on $b^{p}$ for some $m \geq 1$, we first deal with the case $u \in b^{p}\left(H_{\delta}\right)$ for some $\delta>0$. Fix $z \in H$. Letting $w=(x, y)$, we have

$$
\begin{aligned}
\Pi_{m} u(z) & =c_{m} \int_{H} u(w) y^{m} D_{y}^{m} R(z, w) d w \\
& =-2 c_{m} \int_{\partial H} \int_{0}^{\infty} u(x, y) y^{m} D_{y}^{m+1} P(z,(x, y)) d y d x .
\end{aligned}
$$

After integrating by parts in the inner integral in (4.3), we have

$$
\begin{aligned}
\Pi_{m} u(z)= & 2 m c_{m} \int_{\partial H} \int_{0}^{\infty} u(x, y) y^{m-1} D_{y}^{m} P(z,(x, y)) d y d x \\
& +2 c_{m} \int_{\partial H} \int_{0}^{\infty}\left[D_{y} u(x, y)\right] y^{m} D_{y}^{m} P(z,(x, y)) d y d x .
\end{aligned}
$$

The first term above equals $2 u(z)$ from the induction hypothesis. To evaluate the second term, we integrate by parts $m$ times in the inner integral, obtaining

$$
2 c_{m}(-1)^{m} \sum_{j=0}^{m}\left(\begin{array}{c}
m \\
j
\end{array}\right) \frac{m !}{(m-j) !} \int_{\partial H} \int_{0}^{\infty}\left[D_{y}^{m-j+1} u(x, y)\right] y^{m-j} P(z,(x, y)) d y d x .
$$

Hence, (4.4) equals $-u(z)$ from Lemma 4.1, which implies $\Pi_{m} u=u$.

Assuming that $\Pi_{m-1}$ is the identity on $b^{p}$, we have shown that $\Pi_{m} u=u$ whenever $u \in b^{p}\left(H_{\delta}\right)$ for some $\delta>0$. To handle the general $u \in b^{p}$, apply the above to $\tau_{\delta} u$ and let $\delta \rightarrow 0$. This completes the induction and hence the proof that each $\Pi_{m}$ is the identity on every $b^{p}$. 
We now prove the boundedness of $\Pi_{m}$. Because

$$
\left|y^{m} D_{y}^{m} R(z, w)\right| \leq \frac{C}{|z-\bar{w}|^{n}},
$$

we can show the boundedness of $\Pi_{m}$ for the range $1<p<\infty$ by the exact same method we used in the proof of Theorem 3.2. Hence, we only need to consider the $p=1$ case.

Let $f \in L^{1}$ and assume $m>0$. Then

$$
\begin{aligned}
\left\|\Pi_{m} f\right\|_{1} & \leq C \int_{H} \int_{H}|f(w)| \frac{y^{m}}{|z-\bar{w}|^{n+m}} d w d z \\
& \leq C \int_{H} \int_{H}|f(w)| \frac{y}{|z-\bar{w}|^{n+1}} d w d z .
\end{aligned}
$$

Therefore by Fubini's Theorem and the estimate

$$
\int_{H} \frac{1}{|z-\bar{w}|^{n+1}} d z \leq \frac{C}{y}
$$

we get $\left\|\Pi_{m} f\right\|_{1} \leq C\|f\|_{1}$ as desired. (Here $C=C(n, m, p)$.)

That operators analogous to $\Pi_{m}$ can be bounded on $L^{1}$ goes back to [1].

Dense subspaces revisited. The boundedness of $\Pi_{m}$ on $L^{p}$ leads easily to the existence of useful dense subspaces of $b^{p}$ for the full range $1 \leq p<\infty$. (Recall that in Section 3 we showed only that the spaces $\mathcal{M}_{\alpha}$ and $b^{p}\left(H_{\delta}\right)$ are dense in $b^{p}$ for the range $1<p<\infty$.) For $m$ a positive integer, we let $\mathcal{D}_{m}$ denote the vector space of functions $u$ harmonic on $H$ that satisfy

$$
|u(z)| \leq \frac{C}{1+|z|^{m}}
$$

for all $z \in H$.

Theorem 4.3. If $1 \leq p<\infty$ and $m>0$, then $\mathcal{D}_{n+m}$ is a dense subspace of $b^{p}$.

Proof. The space $\mathcal{D}_{n+m}$ is clearly contained in $b^{p}$. To prove density, let $u \in b^{p}$. Choose compact sets $K_{1}, K_{2}, \cdots \subset H$ such that $K_{j} \subset K_{j+1}$ and $H=\bigcup K_{j}$. Setting $u_{j}$ equal to $u$ times the characteristic function of $K_{j}$, we easily check that $\Pi_{m} u_{j} \in \mathcal{D}_{n+m}$ from (3.8). Because

$$
\left\|\Pi_{m} u_{j}-u\right\|_{p}=\left\|\Pi_{m}\left(u_{j}-u\right)\right\|_{p} \leq\left\|\Pi_{m}\right\|\left\|u_{j}-u\right\|_{p} \rightarrow 0
$$

as $j \rightarrow \infty$, we have $\mathcal{D}_{n+m}$ dense in $b^{p}$ as desired.

Some results and references on harmonic approximation in the $L^{1}$-norm for more general domains can be found in [8].

Derivative norms on $b^{p}$. We now prove a useful result that relates the Bergman norm to a "derivative norm". Here (and in the rest of the paper) the expression $A(f) \approx B(f)$ means that there are two positive constants $c$ and $C$ such that the nonnegative quantities $A(f)$ and $B(f)$ satisfy

$$
c A(f) \leq B(f) \leq C A(f)
$$

for all $f$ under consideration. 
Theorem 4.4. If $1 \leq p<\infty$ and $m \geq 0$, then

$$
\|u\|_{p} \approx \sum_{|\alpha|=m}\left\|y^{m} D^{\alpha} u\right\|_{p} \approx\left\|y^{m} D_{y}^{m} u\right\|_{p}
$$

as $u$ ranges over $b^{p}$.

Note the proviso "as $u$ ranges over $b^{p}$ " in Theorem 4.4; the norm equivalence stated there would fail if we allowed $u$ to vary over all harmonic functions on $H$.

To prove Theorem 4.4, we only need to show that there are positive constants $C_{1}, C_{2}$ (depending only on $n, m, p$ ) such that

$$
C_{1} \sum_{|\alpha|=m}\left\|y^{m} D^{\alpha} u\right\|_{p} \leq\|u\|_{p} \leq C_{2}\left\|y^{m} D_{y}^{m} u\right\|_{p}
$$

for all $u \in b^{p}$. We prove (4.7) one inequality at a time.

Theorem 4.5. If $1 \leq p<\infty$ and $m \geq 0$, then there is a positive constant $C=$ $C(n, m, p)$ such that

$$
\sum_{|\alpha|=m}\left\|y^{m} D^{\alpha} u\right\|_{p} \leq C\|u\|_{p}
$$

for all $u \in b^{p}$.

Proof. Let $u \in b^{p}$ and let $\alpha$ be a multi-index with $|\alpha|=m>0$ (if $m=0$, then (4.8) is clearly true). Then by Theorem 4.2 ,

$$
\Pi_{1} u(z)=u(z)=-2 \int_{H} u(w) w_{n} D_{w_{n}} R(z, w) d w
$$

for $z \in H$. Differentiating through the integral in (4.9) and using the estimate (3.8), we have

$$
\left|D^{\alpha} u(z)\right| \leq C \int_{H}|u(w)| \frac{w_{n}}{|w-\bar{z}|^{n+m+1}} d w .
$$

Let $p=1$. Writing $z=(x, y)$, we see from (4.10) that

$$
\begin{aligned}
\left\|y^{m} D^{\alpha} u\right\|_{1} & \leq C \int_{H} y^{m} \int_{H}|u(w)| \frac{w_{n}}{|w-\bar{z}|^{n+m+1}} d w d z \\
& \leq C \int_{H}|u(w)| w_{n} \int_{H} \frac{1}{|w-\bar{z}|^{n+1}} d z d w . \\
& =C\|u\|_{1} .
\end{aligned}
$$

This proves (4.8) in the $p=1$ case.

Now let $1<p<\infty$. Starting with (4.10), we apply Hölder's inequality to the functions

$$
|u(w)|\left(\frac{w_{n}}{|w-\bar{z}|^{n+m+1}}\right)^{1 / p},\left(\frac{w_{n}}{|w-\bar{z}|^{n+m+1}}\right)^{1 / q}
$$

(here $q$ denotes the index conjugate to $p$ ). With $z=(x, y)$, we then obtain

$$
\begin{aligned}
\left|D^{\alpha} u(z)\right|^{p} & \leq C\left(\int_{H}|u(w)|^{p} \frac{w_{n}}{|w-\bar{z}|^{n+m+1}} d w\right)\left(\int_{H} \frac{w_{n}}{|w-\bar{z}|^{n+m+1}} d w\right)^{p / q} \\
& \leq C\left(\int_{H}|u(w)|^{p} \frac{w_{n}}{|w-\bar{z}|^{n+m+1}} d w\right) \frac{1}{y^{m p / q}} .
\end{aligned}
$$


Thus

$$
\begin{aligned}
\left\|y^{m} D^{\alpha} u\right\|_{p}^{p} & \leq C \int_{H}|u(w)|^{p} w_{n} \int_{H} \frac{y^{m}}{|w-\bar{z}|^{n+m+1}} d z d w \\
& =C\|u\|_{p}^{p},
\end{aligned}
$$

completing the proof.

For the other inequality in (4.7), we will use the identity given in the next lemma.

Lemma 4.6. If $1 \leq p<\infty$ and $u \in b^{p}$, then

$$
\Pi_{m}\left(y^{k} D_{y}^{k} u\right)(z)=\frac{c_{m}}{c_{m+k}} u(z)
$$

for all $m, k \geq 0$ and for every $z \in H$.

Proof. Fix $z \in H$; note that the left side of (4.11) is well defined by Theorem 4.5. Assume that $u \in b^{p}\left(H_{\delta}\right)$ for some $\delta>0$, and fix $k \geq 0$. With $w=(x, y)$, we have

$$
\Pi_{0}\left(y^{k} D_{y}^{k} u\right)(z)=-2 \int_{\partial H} \int_{0}^{\infty}\left[D_{y}^{k} u(x, y)\right] y^{k} D_{y} P(z,(x, y)) d y d x .
$$

After integrating by parts in the inner integral above, we obtain

$$
\begin{aligned}
\Pi_{0}\left(y^{k} D_{y}^{k} u\right)(z)= & 2 \int_{\partial H} \int_{0}^{\infty}\left[D_{y}^{k+1} u(x, y)\right] y^{k} P(z,(x, y)) d y d x \\
& +2 k \int_{\partial H} \int_{0}^{\infty}\left[D_{y}^{k} u(x, y)\right] y^{k-1} P(z,(x, y)) d y d x \\
= & \frac{1}{c_{k}} u(z),
\end{aligned}
$$

where we have used Lemma 4.1. For this $u$ we have shown that (4.11) holds for all $k$ in the $m=0$ case.

Proceeding with this $u$ by induction on $m$, suppose (4.11) holds for all $k$ with $m-1$ in place of $m$. For any $k$ we then have

$$
\Pi_{m}\left(y^{k} D_{y}^{k} u\right)(z)=c_{m} \int_{\partial H} \int_{0}^{\infty}\left[D_{y}^{k} u(x, y)\right] y^{m+k} D_{y}^{m} R(z,(x, y)) d y d x .
$$

After integration by parts in the inner integral, we arrive at

$$
\begin{aligned}
\Pi_{m}\left(y^{k} D_{y}^{k} u\right)(z) & =\frac{c_{m}}{c_{m-1}}\left(\Pi_{m-1}\left(y^{k+1} D_{y}^{k+1} u\right)(z)+(m+k) \Pi_{m-1}\left(y^{k} D_{y}^{k} u\right)(z)\right) \\
& =\frac{c_{m}}{c_{m+k}} u(z),
\end{aligned}
$$

where we have used the induction hypothesis. We are done in the case where $u \in b^{p}\left(H_{\delta}\right)$ for some $\delta>0$.

For an arbitrary $u \in b^{p}$, Theorem 4.5 and the $L^{p}$-boundedness of $\Pi_{m}$ show that we can make our usual limiting argument for $\tau_{\delta} u$ as $\delta \rightarrow 0$ to obtain (4.11) for $u$. The proof of the lemma is complete.

The following theorem completes the proof of Theorem 4.4.

Theorem 4.7. If $1 \leq p<\infty$ and $m \geq 0$, then there is a positive constant $C=$ $C(n, m, p)$ such that

for all $u \in b^{p}$.

$$
\|u\|_{p} \leq C\left\|y^{m} D_{y}^{m} u\right\|_{p}
$$


Proof. If $u \in b^{p}$, then $y^{m} D_{y}^{m} u \in L^{p}$ by Theorem 4.5. Hence by Lemma 4.6 and Theorem 4.2, we have

$$
\|u\|_{p}=\left\|\left(c_{m+1} / c_{1}\right) \Pi_{1}\left(y^{m} D_{y}^{m} u\right)\right\|_{p} \leq C\left\|y^{m} D_{y}^{m} u\right\|_{p}
$$

for all $u \in b^{p}$.

Remarks. 1. The norm equivalence expressed in Theorem 4.4 suggests that the harmonic Bloch space (defined in Section 5) - rather than the space of bounded harmonic functions - is the "right" limit of $b^{p}$ as $p \rightarrow \infty$. 2. Sheldon Axler pointed out to us that some of the material in this section is analogous to 4.2.7-4.2.10 in $[12]$.

\section{The harmonic Bloch Space And the Dual SPaCe of $b^{1}$}

In this section we identify the dual space of $b^{1}$. To motivate some of the definitions to come, suppose $\Lambda \in\left(b^{1}\right)^{*}$. By the Hahn-Banach Theorem, there is a function $f \in L^{\infty}$ such that

$$
\Lambda(u)=\int_{H} u f d V
$$

for all $u \in b^{1}$. Now $u=\Pi u$, and so we could hope that

$$
\Lambda(u)=\int_{H}(\Pi u) f d V=\int_{H} u(\Pi f) d V
$$

for all such $u$. Unfortunately, $\Pi f$ is not always well-defined for $f \in L^{\infty}$ (recall $R(z, \cdot)$ is not in $\left.L^{1}\right)$. However, note that the $b^{1}$-cancellation property (Theorem 2.2 ) implies that constants will play no role in the dual space of $b^{1}$. Thus, if we expect the dual space of $b^{1}$ to be a space of functions on $H$, it must be a space of functions that vanish at some prescribed point of $H$. This leads to a natural modification of the operator $\Pi$, as we now describe.

We first set $z_{0}=(0,1)$. For $z, w \in H$, define

$$
\tilde{R}(z, w)=R(z, w)-R\left(z_{0}, w\right) .
$$

Because of cancellation at infinity, the kernel $\tilde{R}(z, \cdot)$ belongs to $L^{1}$ (as we show below). Thus we can define $\tilde{\Pi}$ on $L^{\infty}$ by

$$
\tilde{\Pi} f(z)=\int_{H} f(w) \tilde{R}(z, w) d w .
$$

As we shall see, $\tilde{\Pi}$ maps $L^{\infty}$ into the harmonic Bloch space. A harmonic function $u$ on $H$ is called a Bloch function if

$$
\|u\|_{\mathcal{B}}=\sup y|\nabla u(x, y)|<\infty
$$

where the supremum is taken over all $(x, y) \in H$ and $\nabla u$ denotes the gradient of $u$. (If $u$ is complex valued, then we use the $\mathbf{C}^{n}$-norm to calculate $|\nabla u|$.) We let $\mathcal{B}$ denote the collection of Bloch functions on $H$ and let $\tilde{\mathcal{B}}$ denote the subspace of functions in $\mathcal{B}$ that vanish at $z_{0}$. The space $\tilde{\mathcal{B}}$ is a Banach space under the Bloch norm \|\|$_{\mathcal{B}}$.

To show that $\tilde{\Pi}$ maps $L^{\infty}$ into $\mathcal{B}$ (actually onto $\tilde{\mathcal{B}}$ ), we need an estimate on $\tilde{R}(z, w)$. We obtain this via two inequalities for real numbers (one of which is not 
so obvious). We start with the easier one: If $m$ is a positive integer, then for any $b, d>0$,

$$
\begin{aligned}
\left|\frac{1}{b^{m}}-\frac{1}{d^{m}}\right| & =\frac{\left|b^{m}-d^{m}\right|}{b^{m} d^{m}} \\
& \leq \frac{\left|m(b-d)\left(b^{m-1}+d^{m-1}\right)\right|}{b^{m} d^{m}} \\
& \leq m|b-d|\left(\frac{1}{b^{m} d}+\frac{1}{b d^{m}}\right)
\end{aligned}
$$

where we used the Mean Value Theorem in (5.2). The second inequality we need is given in the following lemma.

Lemma 5.1. For each positive integer $m$, there is a constant $C=C(m)$ such that if $0<a \leq b$ and $0<c \leq d$, then

$$
\left|\frac{a^{2}}{b^{m+2}}-\frac{c^{2}}{d^{m+2}}\right| \leq C(|a-c|+|b-d|)\left(\frac{1}{b^{m} d}+\frac{1}{b d^{m}}\right) .
$$

Proof. Without loss of generality, we may assume that $b \leq d$. Let $B=b^{(m+2) / 2}$ and $D=d^{(m+2) / 2}$. Then the left side of our inequality equals

$$
\left|\frac{a^{2}}{B^{2}}-\frac{c^{2}}{D^{2}}\right|=\left(\frac{a}{B}+\frac{c}{D}\right)\left|\frac{a}{B}-\frac{c}{D}\right|
$$

Note that

$$
\frac{a}{B}+\frac{c}{D} \leq \frac{1}{b^{m / 2}}+\frac{1}{d^{m / 2}} \leq \frac{2}{b^{m / 2}} .
$$

Therefore, after subtracting and adding $a / D$ within the absolute values on the right of (5.4), we see that (5.4) is less than or equal to

$$
\frac{2 a}{b^{m / 2}}\left|\frac{1}{B}-\frac{1}{D}\right|+\frac{2|a-c|}{b^{m / 2} D} .
$$

Let $I$ and $I I$ denote, respectively, the two summands of (5.5). From the Mean Value Theorem,

$$
d^{(m+2) / 2}-b^{(m+2) / 2} \leq \frac{m+2}{2}(d-b) d^{m / 2} .
$$

Therefore,

$$
\begin{aligned}
I & \leq(m+2)|b-d| \frac{a}{b^{m / 2}} \frac{d^{m / 2}}{b^{(m+2) / 2} d^{(m+2) / 2}} \\
& \leq(m+2)|b-d| \frac{1}{b^{m} d} .
\end{aligned}
$$

Similarly,

$$
\begin{aligned}
I I & =2|a-c| \frac{1}{b^{m / 2} d^{m / 2} d} \\
& \leq 2|a-c| \frac{1}{b^{m} d} .
\end{aligned}
$$

The conclusion is immediate from (5.6) and (5.7).

The following is an easy consequence of the above inequalities. 
Theorem 5.2. There is a constant $C=C(n)$ such that

$$
|\tilde{R}(z, w)| \leq C\left|z-z_{0}\right|\left(\frac{1}{|z-\bar{w}|^{n}\left|z_{0}-\bar{w}\right|}+\frac{1}{|z-\bar{w}|\left|z_{0}-\bar{w}\right|^{n}}\right)
$$

for all $z, w \in H$.

Proof. From (3.2), we can easily check that $|\tilde{R}(z, w)|$ is bounded by a constant times

$$
\left|\frac{\left(z_{n}+w_{n}\right)^{2}}{|z-\bar{w}|^{n+2}}-\frac{\left(1+w_{n}\right)^{2}}{\left|z_{0}-\bar{w}\right|^{n+2}}\right|+\left|\frac{1}{|z-\bar{w}|^{n}}-\frac{1}{\left|z_{0}-\bar{w}\right|^{n}}\right|
$$

for $z, w \in H$. On the first summand we use Lemma 5.1 (note that $\left|z_{n}-1\right| \leq\left|z-z_{0}\right|$ ), while we use (5.3) on the second summand. The theorem now follows easily.

Theorem 5.2 implies that $\tilde{R}(z, \cdot)$ belongs to $L^{1}$ for each fixed $z \in H$. Thus, if $f \in L^{\infty}$, then $\tilde{\Pi} f$ is well defined on $H$ by (5.1); furthermore $\tilde{\Pi} f$ is a harmonic function on $H$. We next show that $\tilde{\Pi}$ maps $L^{\infty}$ into $\tilde{\mathcal{B}}$.

Theorem 5.3. $\tilde{\Pi}$ is a bounded linear map of $L^{\infty}$ into $\tilde{\mathcal{B}}$.

Proof. Let $f \in L^{\infty}$. Then $\tilde{\Pi} f\left(z_{0}\right)=0$, clearly. For any $j$ we have

$$
\begin{aligned}
\left|z_{n} D_{z_{j}} \tilde{\Pi} f(z)\right| & =z_{n}\left|\int_{H} f(w) D_{z_{j}} R(z, w) d w\right| \\
& \leq C\|f\|_{\infty} z_{n} \int_{H} \frac{1}{|z-\bar{w}|^{n+1}} d w \\
& \leq C\|f\|_{\infty}
\end{aligned}
$$

for all $z \in H$, with $C=C(n)$; we have used the estimate (4.5). This shows $\tilde{\Pi} f \in \tilde{\mathcal{B}}$ with $\|\tilde{\Pi} f\|_{\mathcal{B}} \leq C\|f\|_{\infty}$.

Our main goal in this section is to show $\left(b^{1}\right)^{*} \cong \tilde{\mathcal{B}}$. For this purpose, let $\Lambda \in\left(b^{1}\right)^{*}$. Then by the Hahn-Banach Theorem, there exists $f \in L^{\infty}$ such that

$$
\Lambda u=\int_{H} u f d V
$$

for all $u \in b^{1}$, with $\|\Lambda\|=\|f\|_{\infty}$. Recalling that $u=\Pi u$ for $u \in b^{1}$, we wish to show that

$$
\Lambda u=\int_{H} \Pi u f d V=\int_{H} u \tilde{\Pi} f d V
$$

and thus that $\Lambda$ is given by $\tilde{\Pi} f \in \tilde{\mathcal{B}}$. Unfortunately, the last integral need not be well defined. However, $\Lambda$ is determined by its action on the dense subspace $\mathcal{D}_{n+1} \subset b^{1}$ (see (4.6) for the definition of the spaces $\mathcal{D}_{m}$ ). Furthermore, if $v \in \tilde{\mathcal{B}}$, then

$$
|v(x, y)| \leq 2\|v\|_{\mathcal{B}}(1+|\log y|+2 \log (1+|x|))
$$

(see [2]), so that $u v \in L^{1}$ whenever $u \in \mathcal{D}_{n+1}$.

We will need the next lemma in proving that (5.8) holds for $u \in \mathcal{D}_{n+1}$.

Lemma 5.4. If $u \in \mathcal{D}_{n+1}$, then

$$
\int_{H} \int_{H}|u(z) \tilde{R}(z, w)| d w d z<\infty .
$$


Proof. If $u \in \mathcal{D}_{n+1}$, then $|u(z)| \leq C /\left|z-\bar{z}_{0}\right|^{n+1}$ on $H$. Thus, by (4.6) and Theorem 5.2 , we obtain

$$
\begin{aligned}
|u(z) \tilde{R}(z, w)| & \leq \frac{C}{\mid z-\overline{\left.z_{0}\right|^{n}}}\left(\frac{1}{|z-\bar{w}|^{n}\left|z_{0}-\bar{w}\right|}+\frac{1}{|z-\bar{w}|\left|z_{0}-\bar{w}\right|^{n}}\right) \\
& \leq \frac{C}{\left|z-\overline{z_{0}}\right|^{n}}\left(\frac{1}{|z-\bar{w}|^{n}\left(1+w_{n}\right)}+\frac{1}{\left(z_{n}+w_{n}\right)\left|z_{0}-\bar{w}\right|^{n}}\right) .
\end{aligned}
$$

Using the Poisson-integral device from the proof of Lemma 3.1 we have

$$
\int_{H}|u(z) \tilde{R}(z, w)| d w \leq \frac{C}{\left|z-\overline{z_{0}}\right|^{n}} \int_{0}^{\infty} \frac{1}{\left(z_{n}+w_{n}\right)\left(1+w_{n}\right)} d w_{n} .
$$

The last integral equals $\left(\log z_{n}\right) /\left(z_{n}-1\right)$, which is comparable to

$$
\left(1+\left|\log z_{n}\right|\right) /\left(1+z_{n}\right) .
$$

Using the Poisson-integral device once again we obtain

$$
\int_{H} \int_{H}|u(z) \tilde{R}(z, w)| d w d z \leq C \int_{0}^{\infty} \frac{1+\left|\log z_{n}\right|}{\left(1+z_{n}\right)^{2}} d z_{n}<\infty,
$$

where $C=C(n, u)$.

Theorem 5.5. If $\Lambda \in\left(b^{1}\right)^{*}$, then there is $v \in \tilde{\mathcal{B}}$ such that

$$
\Lambda(u)=\int_{H} u v d V
$$

for all $u \in \mathcal{D}_{n+1}$. Moreover, $\|v\|_{\mathcal{B}} \leq C\|\Lambda\|$, where $C=C(n)$.

Proof. We know that $\Lambda$ is given by some $f \in L^{\infty}$ as in (5.8), with $\|\Lambda\|=\|f\|_{\infty}$. Let $v=\tilde{\Pi} f$. Then by Theorem 5.3,v $\in \tilde{\mathcal{B}}$ and $\|v\|_{\mathcal{B}} \leq C\|f\|_{\infty}=C\|\Lambda\|$. If $u \in \mathcal{D}_{n+1}$, then

$$
\begin{aligned}
\int_{H} u v d V & =\int_{H} u(z) \int_{H} f(w) \tilde{R}(z, w) d w d z \\
& =\int_{H} f(w) \int_{H} u(z) \tilde{R}(z, w) d z d w .
\end{aligned}
$$

(Switching the order of integration above is permissible by Lemma 5.4.) Note that the inner integral in (5.10) equals $u(w)$ by the $b^{1}$-cancellation property. Thus (5.10) equals $\Lambda(u)$, completing the proof.

We next show that every $v \in \tilde{\mathcal{B}}$ induces a bounded linear functional on $b^{1}$. We first review some additional properties of Bloch functions: If $v \in \tilde{\mathcal{B}}$ and $\delta>0$, then

$$
\begin{aligned}
& \left\|\tau_{\delta} v\right\|_{\mathcal{B}} \leq\|v\|_{\mathcal{B}} \\
& \tau_{\delta} v=P[v(\cdot, \delta)] \text { on } H ; \\
& \left|D_{y}^{m} v(x, y)\right| \leq C\|v\|_{\mathcal{B}} / y^{m}, \quad m=1, \ldots
\end{aligned}
$$

Inequality (5.11) is clear, (5.12) is proved in [2], and (5.13), with $C=C(n, m)$, follows from Cauchy's estimates.

We now extend the domain of $\tilde{\Pi}$ to the set of all functions $f$ for which the integrand in (5.1) belongs to $L^{1}$. From (5.9), we then see that $\tilde{\Pi} v$ is harmonic on $H$ whenever $v \in \mathcal{B}$.

The identity expressed in the next lemma will be helpful in finishing our proof that $\left(b^{1}\right)^{*} \cong \tilde{\mathcal{B}}$. (This lemma is the $\tilde{\mathcal{B}}$-analogue of Lemma 4.6.) 
Lemma 5.6. If $v \in \tilde{\mathcal{B}}$, then $v=c_{m} \tilde{\Pi}\left(y^{m} D_{y}^{m} v\right)$ for $m \geq 0$.

Proof. We have already observed that $\tilde{\Pi}\left(y^{m} D_{y}^{m} v\right)$ is well defined when $m=0$; property (5.13) shows that this expression is well defined if $m>0$.

To prove the lemma we start with $v=\tau_{\delta} u$ for some $u \in \tilde{\mathcal{B}}$; note that $v \in \mathcal{B}$, but $v$ need not be in $\tilde{\mathcal{B}}$. For such a function $v$, we follow the strategy of Lemma 4.1. Thus we will show

$$
v(z)-v\left(z_{0}\right)=-2 c_{m} \int_{H}\left[D_{y}^{m+1} v(w)\right] y^{m} \tilde{P}(z, w) d w,
$$

where $\tilde{P}(z, w)=P(z, w)-P\left(z_{0}, w\right)$ and $w=(x, y)$. Note that the integrand in (5.14) belongs to $L^{1}$ by (5.13) and the estimate $|\tilde{P}(z, w)| \leq C /|z-\bar{w}|^{n}$ (harder to prove than (5.3) but easier than Lemma 5.1).

The integral in (5.14) equals

$$
\int_{0}^{\infty} y^{m} \int_{\partial H} D_{y}^{m+1} v(w) \tilde{P}(z+(0, y), x) d x d y .
$$

Because (5.13) implies that $D_{y}^{m+1} v$ is the Poisson integral of its boundary values on every half-space contained in $H$, (5.15) equals

$$
\int_{0}^{\infty} y^{m}\left(D_{y}^{m+1} v(z+(0,2 y))-D_{y}^{m+1} v\left(z_{0}+(0,2 y)\right)\right) d y .
$$

Simple calculus shows that

$$
\left|D_{y}^{m+1} v(z+(0,2 y))-D_{y}^{m+1} v\left(z_{0}+(0,2 y)\right)\right| \leq \frac{C\|v\|_{\mathcal{B}}}{(y+\delta)^{m+2}}\left|z-z_{0}\right| .
$$

The last estimate allows us to integrate by parts $m$ times in (5.16); doing so then establishes (5.14).

Returning to the statement of Theorem 5.6, consider the $m=0$ case. By (4.1),

$$
\tilde{\Pi} v(z)=-2 \int_{\partial H} \int_{0}^{\infty} v(x, y) D_{y} \tilde{P}(z,(x, y)) d y d x .
$$

Integrating by parts in the inner integral, we have

$$
\begin{aligned}
\tilde{\Pi} v(z)= & 2 \int_{\partial H} v(x, 0) \tilde{P}(z, x) d x \\
& +2 \int_{\partial H} \int_{0}^{\infty} D_{y} v(x, y) \tilde{P}(z,(x, y)) d y d x .
\end{aligned}
$$

From (5.12) and the $m=0$ case of (5.14), we see that the last expression equals $v(z)-v\left(z_{0}\right)$.

Summarizing, we have shown that if $v \in \tilde{\mathcal{B}}$, then

$$
\tilde{\Pi}\left(\tau_{\delta} v\right)(z)=\tau_{\delta} v(z)-\tau_{\delta} v\left(z_{0}\right) .
$$

for $\delta>0$. Using (5.9) and letting $\delta \rightarrow 0$, we see that the dominated convergence theorem gives $\tilde{\Pi} v(z)=v(z)$. We are done with the proof of the theorem in the case $m=0$.

To obtain the theorem for $m>0$ we proceed by induction, recalling that Lemma 4.1 has been proved for all $m$. The proof from here is so similar to the analogous passage in the proof of Lemma 4.6 that we omit the details. 
The $m=0$ case of the last theorem shows that $\tilde{\Pi}$ is the identity on $\tilde{\mathcal{B}}$. The $m=1$ case implies that $\tilde{\Pi}$ is a surjection of $L^{\infty}$ onto $\tilde{\mathcal{B}}$.

We can now show that every $v \in \tilde{\mathcal{B}}$ induces a bounded linear functional on $\mathcal{D}_{n+1}$, hence on $b^{1}$.

Theorem 5.7. If $v \in \tilde{\mathcal{B}}$, then the map $\Lambda$ defined by

$$
\Lambda(u)=\int_{H} u v d V
$$

is a bounded linear functional on $\mathcal{D}_{n+1}$ (with respect to the $b^{1}$-norm). Moreover, $\|\Lambda\| \leq 2\|v\|_{\mathcal{B}}$.

Proof. The linear map $\Lambda$ is well-defined by (5.9). From the $m=1$ case of Lemma 5.6 , we have $v=-2 \tilde{\Pi}\left(y D_{y} v\right)$. Hence,

$$
\int_{H} u v d V=-2 \int_{H} u(z) \int_{H}\left[D_{y} v(w)\right] y \tilde{R}(z, w) d w d z,
$$

where $w=(x, y)$. Because $\left\|y D_{y} v\right\|_{\infty} \leq\|v\|_{\mathcal{B}}$, Lemma 5.4 allows us to switch the order of integration in (5.17), giving

$$
-2 \int_{H}\left[D_{y} v(w)\right] y \int_{H} u(z) \tilde{R}(z, w) d z d w=-2 \int_{H}\left[D_{y} v(w)\right] y u(w) d w,
$$

where we have again used the $b^{1}$-cancellation property. We therefore have

$$
\begin{aligned}
\left|\int_{H} u v d V\right| & \leq 2 \int_{H}\left|D_{y} v(w)\right| y|u(w)| d w \\
& \leq 2\|v\|_{\mathcal{B}}\|u\|_{1},
\end{aligned}
$$

completing the proof.

By combining Theorem 5.5 and Theorem 5.7, we obtain the main result of this section.

Theorem 5.8. $\left(b^{1}\right)^{*} \cong \tilde{\mathcal{B}}$.

Proof. We have a map $\Phi: \tilde{\mathcal{B}} \rightarrow\left(b^{1}\right)^{*}$ given by $v \longmapsto \Lambda_{v}$, where $\Lambda_{v}$ is the map induced by $v$ as in Theorem 5.7. That theorem shows the linear map $\Phi$ is well defined and bounded, while Theorem 5.5 shows that $\Phi$ is onto. To complete the proof, we only need to show $\Phi$ is one-to-one (by the open mapping theorem).

For this purpose, suppose $\Lambda_{v}$ is the zero functional on $b^{1}$ for some $v \in \tilde{\mathcal{B}}$. Because $\tilde{R}(z, \cdot) \in \mathcal{D}_{n+1}$, the $m=0$ case of Lemma 5.6 shows

$$
0=\Lambda_{v}(\tilde{R}(z, \cdot))=\tilde{\Pi} v(z)=v(z)
$$

for every $z \in H$. This implies $\Phi$ is one-to-one, as desired.

Dense subspaces of $b^{1}$. Because $\left(b^{1}\right)^{*} \cong \tilde{\mathcal{B}}$, we can now understand when the subspaces $\mathcal{M}_{\alpha}$ and $b^{1}\left(H_{\delta}\right)$ are dense in $b^{1}$. (The analogous density problems for $b^{p}$, $1<p<\infty$, were discussed in Section 3; see Theorems 3.4 and 3.5.) The next two propositions are relevant to the case of $\mathcal{M}_{\alpha}$.

Proposition $5.9(n=2)$. If $v \in \tilde{\mathcal{B}}$, then $D^{\alpha} v \equiv 0$ if and only if $v \equiv 0$. 
Proof. One direction is clear. For the other direction, we proceed by induction on $|\alpha|$; there is nothing to prove when $\alpha=0$. Assuming the proposition holds for multi-indices of order $m$, suppose $D_{j} D^{\alpha} v \equiv 0$, where $|\alpha|=m$. Then $D^{\alpha} v$ depends on only one variable. But by $(5.13), D^{\alpha} v$ is "Bloch" on each proper half-space of $H_{2}$. Because a Bloch function depending on only one variable is constant, we see that $D^{\alpha} v$ is constant on $\mathrm{H}_{2}$. Using (5.13) again, we see that this constant must be zero. By our induction hypothesis, $v \equiv 0$, completing the proof.

Proposition $5.10(n>2)$.

a. If $v \in \tilde{\mathcal{B}}$ and $\alpha_{n}=|\alpha|$, then $D^{\alpha} v \equiv 0$ if and only if $v \equiv 0$.

b. If $|\alpha|>\alpha_{n}$, then there is a nonzero $v \in \tilde{\mathcal{B}}$ such that $D^{\alpha} v \equiv 0$.

Proof. To prove (a), note that because every derivative of a Bloch function tends to zero at infinity in the " $y$ directions", any harmonic Bloch function that does not depend on $y$ must be constant. A simple induction argument now finishes the proof.

To prove (b), note that if $|\alpha|>\alpha_{n}$, then $\alpha_{j} \neq 0$ for some $j \neq n$. Letting $v$ denote any nontrivial function in $\tilde{\mathcal{B}}$ that is independent of the $j^{\text {th }}$ coordinate variable, we arrive at a nonzero $v \in \tilde{\mathcal{B}}$ with $D^{\alpha} v \equiv 0$.

\section{Corollary 5.11.}

a. If $n=2$, then $\mathcal{M}_{\alpha}$ is dense in $b^{1}$ for all $\alpha \neq 0$.

b. If $n>2$, then $\mathcal{M}_{\alpha}$ is dense in $b^{1}$ if and only if $|\alpha|=\alpha_{n}>0$.

Proof. If $\alpha$ is a nonzero multi-index, then $v \in \tilde{\mathcal{B}} \cong\left(b^{1}\right)^{*}$ vanishes on $\mathcal{M}_{\alpha} \subset \mathcal{D}_{n+1}$ if and only if

$$
0=\int_{H} D_{z}^{\alpha} R(z, w) v(w) d w=D^{\alpha} \int_{H} \tilde{R}(z, w) v(w) d w=D^{\alpha} v(z)
$$

for all $z \in H$. The proof now follows easily from the Hahn-Banach Theorem and the last two propositions.

We now show that $b^{1}\left(H_{\delta}\right)$ is dense in $b^{1}$. As one might expect, Theorem 5.8 is the key, although the proof is different than that of Theorem 3.5.

Theorem 5.12. For each $\delta>0, b^{1}\left(H_{\delta}\right)$ is dense in $b^{1}$.

Proof. Fix $\delta>0$ and $z \in H$. Suppose $v \in \tilde{\mathcal{B}} \cong\left(b^{1}\right)^{*}$ and that the corresponding linear functional vanishes on $b^{1}\left(H_{\delta}\right)$. For nonzero $\alpha, D_{z}^{\alpha} R(z, \cdot) \in \mathcal{D}_{n+1}$, which implies $D_{z}^{\alpha} R(z+(0, \delta), \cdot) \in b^{1}\left(H_{\delta}\right) \cap \mathcal{D}_{n+1}$. Thus

$$
\begin{aligned}
0 & =\int_{H} D_{z}^{\alpha} R(z+(0, \delta), w) v(w) d w \\
& =D_{z}^{\alpha} \int_{H} \tilde{R}(z+(0, \delta), w) v(w) d w \\
& =D^{\alpha} v(z+(0, \delta))
\end{aligned}
$$

for all $\alpha \neq 0$. It follows that the power series of $v$ at the point $z+(0, \delta)$ vanishes except for the constant term. Therefore $v$, being real analytic, is constant on $H$. Because $v \in \tilde{\mathcal{B}}$, we have $v \equiv 0$ on $H$. By a standard corollary of the Hahn-Banach Theorem, $b^{1}\left(H_{\delta}\right)$ must be dense in $b^{1}$. 
Higher-derivative Norms on $\tilde{\mathcal{B}}$. Lemma 5.6 implies the following analogue for the Bloch space of Theorem 4.4.

Theorem 5.13. If $m$ is a positive integer, then

$$
\|v\|_{\mathcal{B}} \approx \sum_{|\alpha|=m}\left\|y^{m} D^{\alpha} v\right\|_{\infty} \approx\left\|y^{m} D_{y}^{m} v\right\|_{\infty}
$$

as $v$ ranges over $\tilde{\mathcal{B}}$.

Proof. We only need to show that there are two positive constants $C_{1}, C_{2}$ (depending only on $m$ and $n$ ) such that

$$
C_{1} \sum_{|\alpha|=m}\left\|y^{m} D^{\alpha} v\right\|_{\infty} \leq\|v\|_{\mathcal{B}} \leq C_{2}\left\|y^{m} D_{y}^{m} v\right\|_{\infty}
$$

for all $v \in \tilde{\mathcal{B}}$. The first inequality is a simple consequence of Cauchy's estimates and the definition of the Bloch norm. To prove the second inequality, let $v \in \tilde{\mathcal{B}}$ and assume $\alpha$ is a multi-index with $|\alpha|=m$. With $w=(x, y)$, Lemma 5.6 implies

$$
v(z)=c_{m} \int_{H} y^{m} D_{y}^{m} v(w) \tilde{R}(z, w) d w
$$

for $z \in H$. Differentiating through the integral and using estimate (3.8), we obtain

$$
\begin{aligned}
\left|z_{n} D_{j} v(z)\right| & \leq C z_{n} \int_{H}\left|y^{m} D_{y}^{m} v(w) D_{z_{j}} R(z, w)\right| d w \\
& \leq C z_{n}\left\|y^{m} D_{y}^{m} v\right\|_{\infty} \int_{H} \frac{d w}{|z-\bar{w}|^{n+1}} \\
& \leq C\left\|y^{m} D_{y}^{m} v\right\|_{\infty},
\end{aligned}
$$

where $C$ depends only on $m$ and $n$. This shows $\|v\|_{\mathcal{B}} \leq C\left\|y^{m} D_{y}^{m} v\right\|_{\infty}$, completing the proof.

\section{HARMONiC CONJUGATES}

Given a harmonic function $u$ on $H$, the functions $v_{1}, \ldots, v_{n-1}$ on $H$ are called harmonic conjugates of $u$ if

$$
\left(v_{1}, \ldots, v_{n-1}, u\right)=\nabla f
$$

for some harmonic function $f$ on $H$. If (6.1) holds, then $v_{1}, \ldots, v_{n-1}$ are partial derivatives of a harmonic function, so they are harmonic on $H$. Also, (6.1) and the condition that $f$ be harmonic is equivalent to the following "generalized CauchyRiemann equations":

$$
\begin{gathered}
D_{k} v_{j}=D_{j} v_{k} ; D_{n} v_{j}=D_{j} u, \\
\sum_{j=1}^{n-1} D_{j} v_{j}+D_{n} u=0 .
\end{gathered}
$$

In particular, if $n=2$, the pair of harmonic functions $(v, u)$ is a conjugate system if and only if $u+i v$ is holomorphic on $\mathrm{H}_{2}$.

If $u$ is harmonic on $H$, then harmonic conjugates of $u$ always exist. Unfortunately, they are far from unique. (When $n>2$, harmonic conjugates for a given $u$ may well differ by more than a constant.) We refer to [2] for more on this. 
Below we show that if $1 \leq p<\infty$ and $u \in b^{p}$, then there are unique harmonic conjugates $v_{1}, \ldots, v_{n-1}$ of $u$ belonging to $b^{p}$. Furthermore, $\left\|v_{j}\right\|_{p} \leq C\|u\|_{p}$ for some constant $C=C(n, p)$.

Note that we exclude the $p=\infty$ case. To see why, consider

$$
u(x, y)=\sum_{j=1}^{n-1} \arctan \left(x_{j} / y\right)
$$

which is bounded and harmonic on $H$. Then a straightforward computation shows that every harmonic conjugate $v_{j}$ of $u$ is of the form $v_{j}(x, y)=\log \left(x_{j}^{2}+y^{2}\right)^{1 / 2}+$ $w_{j}(x)$, so that $v_{j}(x, y) \rightarrow \infty$ as $y \rightarrow \infty$ for each fixed $x$. Thus none of the conjugate functions of $u$ is bounded. (The natural conjugates of bounded harmonic functions are Poisson integrals of BMO-functions; see [5].)

On the other hand, note that we get to include the case $p=1$, in contrast to the Hardy space theory (recall that the Riesz transforms are not bounded on $L^{1}(\partial H)$ ). That conjugation can be bounded on Bergman spaces for $p \leq 1$ was first observed in [7]. (We thank Peter Duren and Joel Shapiro for this reference; see also [3], [6], and [12].) We do not know if our conjugation results for $H$ extend to the range $0<p<1$.

Theorem 6.1. Let $p \in[1, \infty)$ and $u \in b^{p}$. Then there are unique harmonic conjugates $v_{1}, \ldots, v_{n-1}$ of $u$ on $H$ such that $v_{j} \in b^{p}$. Moreover,

$$
\|u\|_{p} \approx \sum_{j=1}^{n-1}\left\|v_{j}\right\|_{p}
$$

Proof. For each fixed $z \in H$, the function $w_{n} D_{w_{j}} R(z, w)$ belongs to $L^{q}$ for all $q>1$. Thus, for $j=1, \ldots, n-1$, we may define

$$
v_{j}(z)=2 \int_{H} u(w) w_{n} D_{w_{j}} R(z, w) d w
$$

for $z \in H$; clearly each $v_{j}$ is harmonic on $H$. (Note the similarity between the definition of $v_{j}$ and $\Pi_{1} u$.) Recalling that

$$
D_{w_{j}} R(z, w)=-D_{z_{j}} R(z, w)
$$

for $j=1, \ldots, n-1$, we can differentiate through the integral above to obtain $D_{k} v_{j}=D_{j} v_{k}$, for $j, k=1, \ldots, n-1$. Also,

$$
D_{n} v_{j}(z)=D_{z_{j}}\left(-2 \int_{H} u(w) w_{n} D_{z_{n}} R(z, w) d w\right) .
$$

Because $D_{z_{n}} R(z, w)=D_{w_{n}} R(z, w)$, we see that $D_{n} v_{j}=D_{j} \Pi_{1} u=D_{j} u$. Finally,

$$
\sum_{j=1}^{n-1} D_{j} v_{j}(z)+D_{n} u(z)=-2 \int_{H} u(w) y \Delta_{z} R(z, w) d w \equiv 0 .
$$

It follows that $v_{1}, \ldots, v_{n-1}$ are harmonic conjugates of $u$.

Referring to the proof of Theorem 4.2, we obtain the estimate $\left\|v_{j}\right\|_{p} \leq C\|u\|_{p}$ in the same way that we proved $\Pi_{1}$ was bounded in the $L^{p}$-norm, noting that $\left|D_{z_{j}} R(z, w)\right|$ satisfies the same estimate as was used on $\left|D_{w_{n}} R(z, w)\right|$. For the 
other inequality, we use Theorem 4.4:

$$
\begin{aligned}
\|u\|_{p} & \approx\left\|y D_{y} u\right\|_{p}=\left\|y\left(\sum_{j=1}^{n-1} D_{j} v_{j}\right)\right\|_{p} \\
& \leq \sum_{j=1}^{n-1}\left\|y\left(D_{j} v_{j}\right)\right\|_{p} \leq C \sum_{j=1}^{n-1}\left\|v_{j}\right\|_{p} .
\end{aligned}
$$

For uniqueness, suppose $u_{1}, \ldots, u_{n-1}$ are also harmonic conjugates of $u$ such that $u_{j} \in b^{p}$ for each $j$. Then by Theorem 4.7,

$$
\left\|v_{j}-u_{j}\right\|_{p} \leq C\left\|z_{n} D_{n}\left(v_{j}-u_{j}\right)\right\|_{p} .
$$

By noticing $D_{n}\left(v_{j}-u_{j}\right)=D_{j}(u-u) \equiv 0$, we have $v_{j}=u_{j}$ as desired.

Let us show that no proper subset of $\left\{v_{1}, \ldots, v_{n-1}\right\}$ will do in $(6.2)$ when $n>2$, at least when $p=2$. For convenience, we will show that the equivalence

$$
\|u\|_{2} \approx \sum_{j=2}^{n-1}\left\|v_{j}\right\|_{2}
$$

fails.

We use an identity that may be of independent interest: If $f \in L^{2}(\partial H)$, then

$$
\|P[f]\|_{2}^{2}=\frac{1}{4 \pi} \int_{\partial H} \frac{|\hat{f}(x)|^{2}}{|x|} d x
$$

here $P[f]$ denotes the Poisson integral of $f$ and $\hat{f}$ is the Fourier transform of $f$ (as defined in $[11])$. To prove $(6.4)$, we view $P[f](x, y)$ as a convolution over $\partial H$ :

$$
P[f](x, y)=P_{y} * f(x)=\int_{\partial H} P_{y}(x-s) f(s) d s,
$$

where the definition of $P_{y}$ should be clear from context. We thus have

$$
\begin{aligned}
\int_{H}|P[f](w)|^{2} d w & =\int_{0}^{\infty} \int_{\partial H}\left|\left(P_{y} * f\right)(x)\right|^{2} d x d y \\
& =\int_{0}^{\infty} \int_{\partial H}\left|\left(P_{y} * f\right) \widehat{(x)}\right|^{2} d x d y \\
& =\int_{0}^{\infty} \int_{\partial H}\left|\hat{P}_{y}(x) \hat{f}(x)\right|^{2} d x d y
\end{aligned}
$$

Now $\hat{P}_{y}(x)=e^{-2 \pi y|x|}$ (see [11], p.16), so we can reverse the order of integration in the last integral to obtain (6.4).

We also need some basic results from Hardy space theory $([10])$ : The map $f \longmapsto$ $R_{j} f$ is bounded on $L^{2}(\partial H)$, where $R_{j} f$ denotes the $j^{\text {th }}$ Riesz transform of $f$. If $f \in L^{2}(\partial H)$, then

$$
\left(R_{j} \hat{f)(x)}=-i \frac{x_{j}}{|x|} \hat{f}(x)\right.
$$

on $\partial H$; furthermore the functions $P\left[R_{j} f\right]$ are harmonic conjugates of $P[f]$.

For any $f \in L^{2}(\partial H)$, then, the above discussion shows that if $P[f] \in b^{2}$, then each $P\left[R_{j} f\right] \in b^{2}$, and thus the functions $P\left[R_{j} f\right]$ must be the harmonic conjugates of $P[f]$ specified by Theorem 6.1 . 
To see that (6.3) fails, note that from the above discussion we have

$$
\begin{aligned}
\sum_{j=2}^{n-1}\left\|P\left[R_{j} f\right]\right\|_{2}^{2} & =\frac{1}{4 \pi} \sum_{j=2}^{n-1} \int_{\partial H} \frac{\mid\left(R_{j} f \widehat{\left.f(x)\right|^{2}}\right.}{|x|} d x \\
& =\frac{1}{4 \pi} \int_{\partial H} \frac{\left(|x|^{2}-x_{1}^{2}\right)}{|x|^{2}} \frac{|\hat{f}(x)|^{2}}{|x|} d x .
\end{aligned}
$$

But we can easily choose $f$ such that the last integral is small when compared with the integral in (6.4). It follows that (6.3) fails, as we wished to show.

Tangential derivative norms. Recall that Theorem 4.4 asserts that the Bergman norm is equivalent to a "derivative norm" involving differentiation only in the normal direction. We can now show that the Bergman norm is equivalent to a tangential derivative norm.

Theorem 6.2. If $1 \leq p<\infty$ and $m \geq 0$, then

$$
\sum_{\substack{|\alpha|=m \\ \alpha_{n}=0}}\left\|y^{m} D^{\alpha} u\right\|_{p} \approx\|u\|_{p}
$$

as $u$ ranges over $b^{p}$.

Proof. By Theorem 4.4, we only need to show that there is $C=C(n, m, p)$ such that

$$
\left\|y^{m} D_{y}^{m} u\right\|_{p} \leq C \sum_{\substack{|\alpha|=m \\ \alpha_{n}=0}}\left\|y^{m} D^{\alpha} u\right\|_{p} .
$$

If $u$ is harmonic, then $\Delta^{k} u=0$ for any integer $k>0$, so that

$$
D_{y}^{2 k} u=-\sum_{j_{1}, \ldots, j_{k}=1}^{n-1} D_{j_{1}}^{2} \cdots D_{j_{k}}^{2} u .
$$

This easily implies (6.5) in the case where $m$ is even.

Now suppose $m=2 k+1$ for some $k \geq 0$. Let $u \in b^{p}$, and let $v_{1}, \ldots, v_{n-1}$ be the harmonic conjugates of $u$ specified in Theorem 6.1. Because

$$
D_{y} u=-\sum_{j=1}^{n-1} D_{j} v_{j}
$$

we have

$$
D_{y}^{m} u=-\sum_{j=1}^{n-1} D_{y}^{2 k} D_{j} v_{j} .
$$

From Theorem 4.4 we then obtain

$$
\begin{aligned}
\left\|y^{m} D_{y}^{m} u\right\|_{p} & \leq \sum_{j=1}^{n-1}\left\|y^{m} D_{y}^{2 k} D_{j} v_{j}\right\|_{p} \\
& \leq C \sum_{j=1}^{n-1}\left\|y^{m} D_{y}^{m} v_{j}\right\|_{p} .
\end{aligned}
$$


Because $D_{y} v_{j}=D_{j} u$, we have $D_{y}^{m} v_{j}=D_{y}^{2 k} D_{j} u$. Writing $D_{y}^{2 k}$ as we did above ( $D_{j} u$ is harmonic) then shows

$$
\begin{aligned}
\left\|y^{m} D_{y}^{m} u\right\|_{p} & \leq C \sum_{j=1}^{n-1}\left\|y^{m} D_{y}^{2 k} D_{j} u\right\|_{p} \\
& \leq C \sum_{j=1}^{n-1} \sum_{j_{1}, \ldots, j_{k}=1}^{n-1}\left\|y^{m} D_{j_{1}}^{2} \cdots D_{j_{k}}^{2} D_{j} u\right\|_{p} .
\end{aligned}
$$

This proves (6.5) for odd $m$, completing the proof of the theorem.

Harmonic conjugation on $\tilde{\mathcal{B}}$. The following theorem was proved in [2].

Theorem 6.3. For $u \in \tilde{\mathcal{B}}$, there are unique harmonic conjugates $v_{1}, \ldots, v_{n-1}$ of $u$ on $H$ such that $v_{j} \in \tilde{\mathcal{B}}$. Moreover, there is a constant $C=C(n)$ such that $\left\|v_{j}\right\|_{\mathcal{B}} \leq C\|u\|_{\mathcal{B}}$ for each $j$.

We could prove the last theorem using the ideas of the proof of Theorem 6.1 as a guide; however, the technical details for $\tilde{\mathcal{B}}$ seem more difficult. The following results can be proved in exactly the same way we proved Theorems 6.1 and 6.2. So we omit the proofs.

Theorem 6.4. If $u \in \tilde{\mathcal{B}}$, then

$$
\|u\|_{\mathcal{B}} \approx \sum_{j=1}^{n-1}\left\|v_{j}\right\|_{\mathcal{B}}
$$

where $v_{1}, \ldots, v_{n-1}$ are the harmonic conjugates of $u$ given by Theorem 6.3.

Theorem 6.5. If $m>0$, then

$$
\sum_{\substack{|\alpha|=m \\ \alpha_{n}=0}}\left\|y^{m} D^{\alpha} u\right\|_{\infty} \approx\|u\|_{\mathcal{B}}
$$

as $u$ ranges over $\tilde{\mathcal{B}}$.

We can also show that no proper subset of $\left\{v_{1}, \ldots, v_{n-1}\right\}$ will do in Theorem 6.4 when $n>2$. In fact, proving that

$$
\|u\|_{\mathcal{B}} \approx \sum_{j=2}^{n-1}\left\|v_{j}\right\|_{\mathcal{B}}
$$

fails is much easier than proving (6.3) fails: We simply choose any nontrivial $u \in \tilde{\mathcal{B}}$ that depends only on $x_{1}$ and $y$. We then have

$$
\begin{gathered}
\sum_{j=2}^{n-1}\left\|v_{j}\right\|_{\mathcal{B}} \approx \sum_{j=2}^{n-1}\left\|y D_{y} v_{j}\right\|_{\infty}=\sum_{j=2}^{n-1}\left\|y D_{j} u\right\|_{\infty}=0 . \\
\text { REFERENCES }
\end{gathered}
$$

1. L. Ahlfors, Some remarks on Teichmüller's space of Riemann surfaces, Ann. of Math. 74 (1961), 171-191. MR 34:4480

2. H. Ajmi and W. Ramey, Harmonic Bloch functions on the upper half space (to appear).

3. S. Axler, Bergman spaces and their operators, Surveys of Some Recent Results in Operator Theory, Vol. 1, Pitman Research Notes in Math. 171, Pitman, 1988, pp. 1-50. MR 90b:47048 
4. S. Axler, P. Bourdon and W. Ramey, Harmonic function theory, Springer-Verlag, New York, 1992. MR 93f:31001

5. C. Fefferman and E. Stein, $H^{p}$-spaces of several variables, Acta Math. 129 (1972), 137-193. MR 56:6263

6. F. Forelli and W. Rudin, Projections on spaces of holomorphic functions in balls, Indiana Univ. Math. J. 24 (1974), 593-602. MR 50:10332

7. G. H. Hardy and J. E. Littlewood, Some properties of conjugate functions, J. Reine Angew. Math. 167 (1931), 405-423

8. H. S. Shapiro, Global geometric aspects of Cauchy's problem for the Laplace operator, research report TRITA-MAT-1989-37, Royal Inst. Tech., Stockholm.

9. A. Shields and D. Williams, Bounded projections, duality, and multipliers in spaces of analytic functions, Trans. Amer. Math. Soc. 162 (1971), 287-302. MR 44:790

10. E. Stein, Singular integrals and differentiability properties of functions, Princeton Univ. Press, Princeton, NJ, 1970. MR 44:7280

11. E. Stein and G. Weiss, Fourier analysis on Euclidean spaces, Princeton Univ. Press, Princeton, NJ, 1971. MR 46:4102

12. K. Zhu, Operator theory in function spaces, Marcel Dekker, New York and Basel, 1990. MR 92c: 47031

Department of Mathematics, Michigan State University, East Lansing, Michigan 48824-1027

E-mail address: ramey@math.msu.edu

Global Analysis Research Center, Department of Mathematics, Seoul National UniVersity, SeOul, Korea \#151-742

E-mail address: hsyi@math.snu.ac.kr 